\title{
Diagnostic yield of Franseen and Fork-Tip biopsy needles for endoscopic ultrasound-guided tissue acquisition: a meta-analysis
}

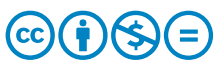

\author{
Authors \\ Muscatiello ${ }^{1}$ \\ Institutions \\ 1 Gastroenterology Unit, University of Foggia, Italy \\ 2 Biostatistics Unit, University of Foggia, Italy \\ 3 AlIMS, Jodhpur, India \\ submitted 15.4.2019 \\ accepted after revision 5.7.2019 \\ Bibliography \\ DOI https://doi.org/10.1055/a-0982-2997 | \\ Endoscopy International Open 2019; 07: E1221-E1230 \\ (c) Georg Thieme Verlag KG Stuttgart · New York \\ eISSN 2196-9736 \\ Corresponding author \\ Antonio Facciorusso, Gastroenterology Unit, Department of \\ Medical Sciences, University of Foggia, AOU Ospedali \\ Riuniti, Viale Pinto, 1, 71100 Foggia, Italy \\ Fax: 00390881733545 \\ antonio.facciorusso@virgilio.it
}

Antonio Facciorusso ${ }^{1,2}$, Valentina Del Prete', Vincenzo Rosario Buccino ${ }^{1}$, Purvi Purohit ${ }^{2,3}$, Puneet Setia ${ }^{2,3}$, Nicola

Supplementary Tables 1 - 5, Supplementary Figs. 1 - 6 Online content viewable at:

https://doi.org/10.1055/a-0982-2997

\section{ABSTRACT}

Background and study aims Although newer needle designs are thought to improve diagnostic outcomes of endo- scopic ultrasound-guided fine-needle biopsy, there is limited evidence on their diagnostic performance. The aim of this meta-analysis was to provide a pooled estimate of the diagnostic performance and safety profile of Franseen and Fork-tip fine-needle biopsy needles.

Patients and methods Computerized bibliographic search on the main databases was performed through March 2019. The primary endpoint was sample adequacy. Secondary outcomes were diagnostic accuracy, optimal histological core procurement, mean number of needle passes, pooled specificity and sensitivity. Safety data were also analyzed.

Results Twenty-four studies with 6641 patients were included and pancreas was the prevalent location of sampled lesions. Overall sample adequacy with the two newer needles was $94.8 \%$ (93.1\%-96.4\%), with superiority of Franseen needle over Fork-tip ( $96.1 \%$ versus $92.4 \%, P<0.001)$. Sample adequacy in targeting pancreatic masses was $95.6 \%$ and both needles produced results superior to fineneedle aspiration (FNA) (odds ratio 4.29, 1.49-12.35 and $1.79,1.01-3.19$ with Franseen and Fork-tip needle, respectively). The rate of histological core procurement was $92.5 \%$, whereas diagnostic accuracy and sensitivity were $95 \%$ and $92.8 \%$, again with no difference between the two needles. Number of needle passes was significantly lower in comparison to FNA (mean difference: -0.42 with Franseen and -1.60 with Fork-tip needle). No significant adverse events were registered.

Conclusion Our meta-analysis speaks in favor of use of newer biopsy needles as a safe and effective tool in endoscopic ultrasound-guided tissue acquisition.

\section{Introduction}

Among the main advantages of endoscopic ultrasound (EUS), tissue acquisition (TA) represents a valuable and accurate diagnostic technique for cytopathological and histological characterization of several abdominal lesions such as pancreatic masses, lymph nodes, or subepithelial lesions (SELs).

In spite of good results observed with EUS-guided fine-needle aspiration (EUS-FNA) and recent developments in this field, such as use of rapid on-site evaluation (ROSE) [1], contrast-enhanced guided FNA or tissue elastography [2], diagnostic sensitivity still remains an issue. Thus, the most important pitfall associated with this procedure is false-negative diagnosis, which has the potential to delay patient care and negatively affect patient outcomes.

Development of EUS-guided fine-needle biopsy (EUS-FNB) needles has generated a great deal of interest in the field of EUS-TA primarily based on proposed advantages over EUS-FNA 
needles concerning diagnostic accuracy. Furthermore, EUS-FNB seems to improve procurement of samples with preserved tissue architecture, thus allowing for immunohistochemistry required for certain diagnoses, obviating ROSE and obtaining results in fewer passes [2].

Two recent meta-analyses reached the conclusion that EUSFNB performed with the reverse bevel needle (ProCore, Cook Medical, Limerick, Ireland) shows comparable diagnostic accuracy and sample adequacy in comparison to EUS-FNA $[3,4]$; even the previous finding of a lower number of passes through the lesion needed to obtain adequate samples with FNB was recently challenged [4].

Two newer FNB needles were recently introduced in endoscopic practice: one with fork-tip design with two leading sharp tips on the opposite side of the lumen (SharkCore, Medtronic, Minneapolis, Minnesota, United States), and another with Franseen tip design with three symmetric cutting edges (Acquire, Boston Scientific Corp, Natick, Massachusetts, United States).

Although these novel needle designs are thought to improve tissue capture and several studies have been published testing these novel devices, there is limited evidence on their diagnostic performance in terms of diagnostic yield and histology core procurement; hence the pressing need to systematically assess the increasing body of evidence in the field to better define their optimal role and safety in endoscopy.

The aim of this meta-analysis was to provide a pooled estimate of the diagnostic performance and safety profile of these two newer needles, namely Franseen and Fork-tip, thus attempting to determine their comparative efficacy and potential utility in EUS-TA.

The primary endpoint was sample adequacy. Secondary outcomes were diagnostic accuracy, optimal histological core procurement, mean number of needle passes, pooled specificity and sensitivity. Safety data were also analyzed.

\section{Patients and methods}

\section{Inclusion and exclusion criteria}

Only studies meeting the following criteria were included: 1) full-text articles recruiting patients undergoing EUS-TA of solid lesions with Franseen or Fork-tip needles; 2) studies published in English; and 3) articles reporting at least one of the following data: sample adequacy, diagnostic accuracy (or data useful for its calculation), histologic core procurement. Case reports, non-endoscopic studies, review articles, and animal models were excluded.

\section{Search strategy}

- Fig. 1 reports the search strategy followed in the meta-analysis.Bibliographic research was conducted on PubMed, EMBASE, Cochrane Library and Google Scholar including all studies fulfilling inclusion criteria published until March 2019. Details of the search strategy adopted are reported in Supplementary Table 1.

Relevant reviews and meta-analyses on use of EUS-TA with newer biopsy needles were examined for potential suitable

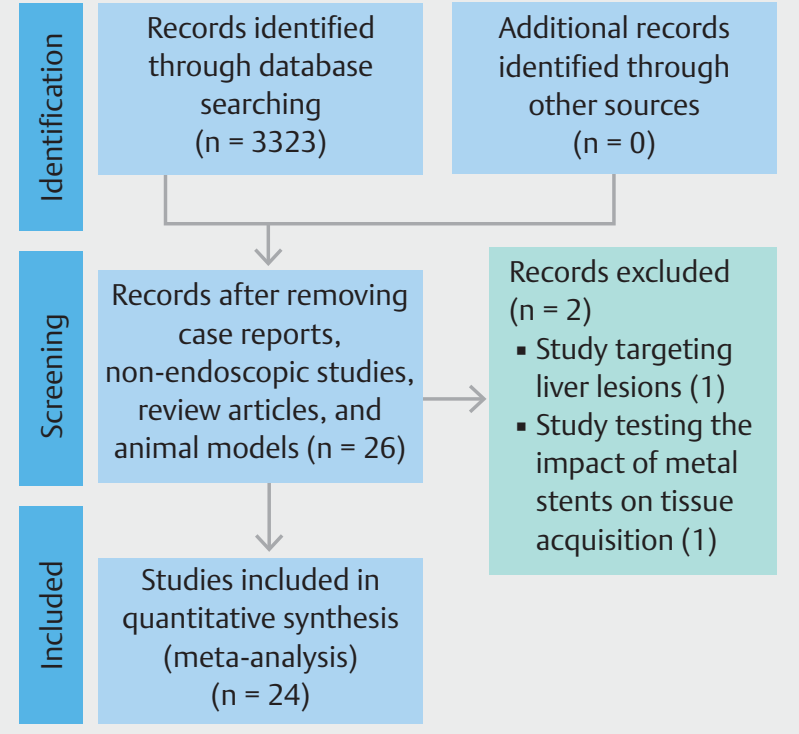

- Fig. 1 Flowchart of included studies.

studies. Authors of included studies were contacted to obtain full text or further information when needed.

Data extraction was conducted by two reviewers (AF and VDP) using a standardized approach (PRISMA Statement). The quality of included studies was assessed by two authors independently (AF, VDP) according to the Cochrane Collaboration's tool for assessing risk of bias [5] for randomized controlled trials (RCTs) and the Newcastle-Ottawa scale [6] for non-randomized studies. Disagreements were solved by discussion and following a third opinion (NM).

\section{Outcomes}

The primary outcome was sample adequacy, defined as ability to procure cytological and/or histological samples adequate for interpretation; secondary outcomes were diagnostic accuracy (defined as true positive + true negative/total number of patients), optimal core histological tissue (defined as samples with high cellularity and quality enabling appropriate core assessment in terms of tissue architecture), specificity (true negative/true negative + false positive), sensitivity (true positive/ true positive + false negative), number of needle passes, and safety.

\section{Statistical analysis}

Diagnostic outcomes were computed overall and then separately pooled for each needle through a random-effects model based on DerSimonian and Laird test, and summary estimates were expressed in terms of rate and 95\% Confidence Interval $(\mathrm{Cl})$. Chi-square and $\mathrm{I}^{2}$ tests were used for across studies comparison of the percentage of variability attributable to heterogeneity beyond chance. $P<0.10$ for chi-square test and $1^{2}<20 \%$ were interpreted as low-level heterogeneity. 
Probability of publication bias was assessed using funnel plots and with Begg and Mazumdar's test.

Safety data were inconsistently reported, hence they were analyzed descriptively.

Pooled diagnostic outcomes of the two needles were compared using the bivariate approach [7].

Sensitivity analysis was conducted according to location of the lesion sampled (pancreas vs lymph nodes vs SEL), quality of included studies, use of ROSE, and study design (single-cohort versus comparative). A separate comparative analysis was conducted with studies directly comparing newer needles to a control device (FNA or reverse-bevel FNB).

All statistical analyses were conducted using RevMan version 5 from the Cochrane collaboration and OpenMeta[Analyst] software. For all calculations a two-tailed $P$ value of less than 0.05 was considered statistically significant.

\section{Results}

\section{Characteristics of included studies}

As shown in - Fig. 1, of 3323 studies initially identified, after preliminary exclusion of papers not fulfilling inclusion criteria, 26 potentially relevant articles were examined. Among these studies, two were excluded because they were focused exclusively on liver lesions [8] or tested the impact of metal stents on tissue acquisition [9].

Finally, 24 studies [10 - 33] with 6641 patients were included in the meta-analysis. Main characteristics of included studies are reported in $>$ Table 1 . Four RCTs were conducted in Asia [14,17-19] and all comparative studies presented two wellbalanced arms in terms of lesion size and clinical-demographical characteristics ( $\triangleright$ Table 1 ). Quality was deemed mainly moderate/high with only three studies assessed as low-quality articles $[21,24,31]$.

Details on methodological characteristics and quality of included articles are shown in Supplementary Table 2.

\section{Sample adequacy}

As reported in > Table 2 and Supplementary Fig. 1, overall sample adequacy with the two newer needles was $94.8 \%$ (93.1\%-96.4\%; $\left.I^{2}=87 \%\right)$. Subanalysis performed according to needle design showed superiority of Franseen needle over Fork-tip (96.1\%, $93.7 \%$ to $98.4 \%$ versus $92.4 \%$, $88.8 \%$ to $96 \%$; $P<0.001)$, although this finding should be interpreted with caution due to the high heterogeneity observed $\left(I^{2}=82.9 \%\right.$ and $88 \%$, respectively; $>$ Table 2 and Supplementary Fig. 2a and Supplementary Fig.2b). There was no evidence of publication bias (data not shown).

The findings of main analysis were confirmed in sensitivity analysis performed according location of sampled lesions, study quality and use of ROSE. Sample adequacy in targeting pancreatic masses was $95.6 \%\left(94 \%-97.3 \%\right.$; $\left.1^{2}=48.9 \%\right)$ with significantly higher rates of adequate samples obtained with Franseen needle (97\%, $94.8 \%$ to $99.3 \%$ versus $92.6 \%, 88.8 \%$ to $96.4 \% ; P=0.006)$. Of note, there was evidence of only lowmoderate heterogeneity in sensitivity analysis for pancreatic masses $\left(I^{2}=13.4 \%\right.$ and $34 \%$, respectively; $>$ Table 2 and $>$ Fig. 2a and $>$ Fig. 2b).

When considering lymph nodes and SELs, findings were in keeping with the aforementioned results (adequacy $97.2 \%$, $94 \%-100 \%$ and $95.6 \%, 92.2 \%-98.9 \%$ with lymph nodes and SELs, respectively) with significant superiority of Franseen over Fork-tip needle (98.4\%, 96\%-100\% versus $77.6 \%$, 46.2\%$100 \%, P<0.001$ for lymph nodes and $97.6 \%, 94 \%-100 \%$ versus $90.5 \%, 79.6 \%-100 \%, P=0.006$ for SELs). Heterogeneity was $0 \%$ and $39 \%$ with the two needles, respectively ( $\triangleright$ Table 2 ).

The above reported results were confirmed in sensitivity analysis restricted to high quality studies and those using ROSE (Supplementary Table 3). As depicted in Supplementary Fig. 3a, five studies [15-19] directly compared Franseen FNB needle to FNA, showing a clear advantage in terms of sample adequacy of Franseen needle (OR 4.29, 1.49-12.35; $P=0.007$ and $\mathrm{I}^{2}=0 \%$ ). Likewise, Fork-tip needle appeared to clearly overperform FNA in the pairwise meta-analysis of 5 studies [23-27] (OR 1.79, 1.01-3.19, $P=0.05$ and $\mathrm{I}^{2}=0 \%$; Supplementary Fig. 3b).

Technical details about handling of the sampled tissue are reported in Supplementary Table 4. Handling protocols were relatively homogeneous with no difference between Franseen and Fork-tip groups.

\section{Optimal histologic core procurement, diagnostic accuracy, sensitivity and specificity}

The rate of histological optimal core procurement obtained with the new FNB needles was $92.5 \%$ (89.8\%-95.3\%) with evidence of high heterogeneity $\left(I^{2}=79.8 \%\right)$. No difference between the two FNB needles was observed neither in main analysis $(93.5 \%, 89.8 \%-97.2 \%$ versus $90.8 \%, 85.5 \%-96.2 \%$; $P=$ 0.4, > Table 2 and Supplementary Fig. 4) nor in subanalysis restricted to pancreatic masses $(94 \%, 89 \%-99 \%$ versus $93.1 \%$, $87.3 \%$ - 99\%; $P=0.7$, $>$ Table 2 ).

Moderate evidence of heterogeneity $\left(I^{2}=33 \%\right.$ to $\left.35 \%\right)$ and no evidence of publication bias was observed, as confirmed with Begg and Mazumdar's test $(P=0.64)$. Overall diagnostic accuracy was $95 \%\left(93.5 \%-96.5 \% ; I^{2}=11 \%\right)$ with no difference between Franseen and Fork-tip needle (95\%, 92.5\%-97.5\% versus $94.4 \%, 92.3 \%-96.5 \% ; P=0.71$, > Table 2 and Supplementary Fig.5). Similarly, subanalysis of pancreatic lesions confirmed the above reported findings (96.8\%, 94.9\%-98.7\% versus $95.2 \%, 92.8 \%-97.6 \% ; P=0.8$ and $\mathrm{I}^{2}=0 \%$ ).

Overall sensitivity of new FNB needles was $92.8 \%, 89.8 \%-$ $95.7 \%$, again with no difference between Franseen and Forktip needles $(93.3 \%, 89.2 \%-97.4 \%$ versus $92.2 \%, 87.9 \%-$ $96.6 \%$, respectively; $P=0.3$, $>$ Table 2 ). Similar results were registered in subanalysis of pancreatic masses ( $>$ Table 2 ). Heterogeneity was low to moderate ( $0 \%$ to $45.6 \%$ ).

As expected, specificity was $100 \%$ with both needles. 
- Table 1 Characteristics of included studies.

\begin{tabular}{|c|c|c|c|c|c|c|c|c|c|}
\hline Study & Needle & $\begin{array}{l}\text { Sam- } \\
\text { ple } \\
\text { size }\end{array}$ & $\begin{array}{l}\text { Study peri- } \\
\text { od/design }\end{array}$ & Country & Age & $\begin{array}{l}\text { Gender } \\
\text { male }\end{array}$ & $\begin{array}{l}\text { Lesion size } \\
(\mathrm{cm})\end{array}$ & Location & ROSE \\
\hline \multicolumn{10}{|l|}{ Franseen } \\
\hline $\begin{array}{l}\text { Adler } \\
2018 \\
{[10]}\end{array}$ & Acquire & 200 & $\begin{array}{l}\text { 2016/Ret- } \\
\text { rospective }\end{array}$ & USA & $63 \pm 14.5$ & $121(60.5 \%)$ & $3.6(0.3-10)$ & $\begin{array}{l}\text { Pancreas: } 55 \% \\
\text { Nodes: } 23 \% \\
\text { SE: } 17 \% \\
\text { Other: } 4 \%\end{array}$ & Yes \\
\hline $\begin{array}{l}\text { Bang } \\
2017 \\
{[11]}\end{array}$ & Acquire & 30 & $\begin{array}{l}\text { 2016/Ret- } \\
\text { rospective }\end{array}$ & USA & $71.5(56-79)$ & $21(70 \%)$ & $3.4(2.8-4)$ & $\begin{array}{l}\text { Pancreas: } 60 \% \\
\text { Nodes: } 10 \% \\
\text { SE: } 16.6 \% \\
\text { Other: } 13.4 \%\end{array}$ & Yes \\
\hline $\begin{array}{l}\text { Haseeb } \\
2018 \\
{[12]}\end{array}$ & Acquire & 132 & $\begin{array}{l}2016 \text { - } \\
2017 / \text { Ret- } \\
\text { rospective }\end{array}$ & USA & $63.5 \pm 13$ & $87(66 \%)$ & NR & $\begin{array}{l}\text { Pancreas: } 73 \% \\
\text { Nodes: } 21 \% \\
\text { SE: } 4 \% \\
\text { Other: } 1 \%\end{array}$ & Yes \\
\hline $\begin{array}{l}\text { Leung Ki } \\
2019 \\
{[13]}\end{array}$ & Acquire & 54 & $\begin{array}{l}\text { 2016/Ret- } \\
\text { rospective }\end{array}$ & France & $70(61-78)$ & $34(74 \%)$ & $2.4 \pm 1.38$ & $\begin{array}{l}\text { Pancreas: } 57 \% \\
\text { Nodes: } 17 \% \\
\text { SE: } 9 \% \\
\text { Other: } 17 \%\end{array}$ & No \\
\hline $\begin{array}{l}\text { Sugiura } \\
2019 \\
{[14]}\end{array}$ & Acquire & 100 & $\begin{array}{l}2017- \\
2018 / \text { Pro- } \\
\text { spective }\end{array}$ & Japan & $70(31-87)$ & $57(57 \%)$ & $2.3(0.7-8.2)$ & $\begin{array}{l}\text { Pancreas: } 81 \% \\
\text { Nodes: } 13 \% \\
\text { SE: } 3 \% \\
\text { Other: } 3 \%\end{array}$ & No \\
\hline \multicolumn{10}{|c|}{ Franseen versus FNA } \\
\hline $\begin{array}{l}\text { Bang } \\
2017 \\
{[15]}\end{array}$ & $\begin{array}{l}\text { Acquire } \\
\text { FNA }\end{array}$ & $\begin{array}{l}46 \\
46\end{array}$ & $\begin{array}{l}\text { NR/Cross- } \\
\text { over RCT }\end{array}$ & USA & $67.9 \pm 14.7$ & $28(60.9 \%)$ & $2.9 \pm 0.8$ & $\begin{array}{l}\text { Pancreas: } \\
100 \%\end{array}$ & Yes \\
\hline $\begin{array}{l}\text { El Hajii } \\
2018 \\
{[16]}\end{array}$ & $\begin{array}{l}\text { Acquire } \\
\text { Expect }\end{array}$ & $\begin{array}{l}51 \\
50\end{array}$ & $\begin{array}{l}2013- \\
2017 / \text { Ret- } \\
\text { rospective }\end{array}$ & USA & NR & NR & $\begin{array}{l}2.98 \\
2.9\end{array}$ & $\begin{array}{l}\text { Pancreas: } 45 \% \\
\text { Pancreas: } 40 \%\end{array}$ & Yes \\
\hline $\begin{array}{l}\text { Fujita } \\
2018 \\
{[17]}\end{array}$ & $\begin{array}{l}\text { Acquire } \\
\text { Expect }\end{array}$ & $\begin{array}{l}17 \\
44\end{array}$ & $\begin{array}{l}2013- \\
2017 / \text { Ret- } \\
\text { rospective }\end{array}$ & Japan & $\begin{array}{l}72(58.5-74.5) \\
67(55-74.8)\end{array}$ & $\begin{array}{l}10(58.8 \%) \\
27(61.3 \%)\end{array}$ & $\begin{array}{l}2.67(1.9-4) \\
2.39(1.6-3)\end{array}$ & $\begin{array}{l}\text { SE: } 100 \% \\
\text { SE: } 100 \%\end{array}$ & No \\
\hline $\begin{array}{l}\text { Ishikawa } \\
2019 \\
{[18]}\end{array}$ & $\begin{array}{l}\text { Acquire } \\
\text { EZShot }\end{array}$ & $\begin{array}{l}50 \\
36\end{array}$ & $\begin{array}{l}2016- \\
\text { 2018/Ret- } \\
\text { rospective }\end{array}$ & Japan & $\begin{array}{l}70.5(60-75) \\
65(57-77)\end{array}$ & $\begin{array}{l}30(60 \%) \\
21(58.3 \%)\end{array}$ & $\begin{array}{l}3(2-3.9) \\
2.3(2.3-3.4)\end{array}$ & $\begin{array}{l}\text { Pancreas: } \\
100 \% \\
\text { Pancreas: } \\
100 \%\end{array}$ & No \\
\hline $\begin{array}{l}\text { Mukai } \\
2018 \\
{[19]}\end{array}$ & $\begin{array}{l}\text { Acquire } \\
\text { Expect }\end{array}$ & $\begin{array}{l}30 \\
30\end{array}$ & $\begin{array}{l}2016 \text { - } \\
\text { 2017/Ret- } \\
\text { rospective }\end{array}$ & Japan & $\begin{array}{l}64.7 \pm 12.5 \\
69 \pm 9.5\end{array}$ & $\begin{array}{l}19(63.3 \%) \\
20(66.6 \%)\end{array}$ & $\begin{array}{l}3 \pm 1.07 \\
2.7 \pm 0.7\end{array}$ & $\begin{array}{l}\text { Pancreas: } \\
100 \% \\
\text { Pancreas: } \\
100 \%\end{array}$ & No \\
\hline \multicolumn{10}{|l|}{ Fork-Tip } \\
\hline $\begin{array}{l}\text { DiMaio } \\
2016 \\
{[20]}\end{array}$ & $\begin{array}{l}\text { Shark- } \\
\text { Core }\end{array}$ & 226 & $\begin{array}{l}2014- \\
\text { 2015/Ret- } \\
\text { rospective }\end{array}$ & USA & $66(18-92)$ & $113(50 \%)$ & $2.6(0.2-15)$ & $\begin{array}{l}\text { Pancreas: } 60 \% \\
\text { Nodes: } 22 \% \\
\text { SE: } 12 \% \\
\text { Other: } 6 \%\end{array}$ & Yes \\
\hline $\begin{array}{l}\text { Ishikawa } \\
2018 \\
{[21]}\end{array}$ & $\begin{array}{l}\text { Shark- } \\
\text { Core }\end{array}$ & 85 & $\begin{array}{l}2015 \text { - } \\
\text { 2016/Ret- } \\
\text { rospective }\end{array}$ & Canada & $62.3 \pm 15$ & $48(60.7 \%)$ & $3.8 \pm 3.7$ & $\begin{array}{l}\text { Pancreas: } 56 \% \\
\text { Nodes: } 15 \% \\
\text { SE: } 26 \% \\
\text { Other: } 3 \%\end{array}$ & No \\
\hline $\begin{array}{l}\text { Larsen } \\
2018 \\
{[22]}\end{array}$ & $\begin{array}{l}\text { Shark- } \\
\text { Core }\end{array}$ & 41 & $\begin{array}{l}2015 \text { - } \\
\text { 2016/Pro- } \\
\text { spective }\end{array}$ & Denmark & $68 \pm 11$ & $22(54 \%)$ & $2.8 \pm 1.1$ & $\begin{array}{l}\text { Pancreas: } \\
100 \%\end{array}$ & No \\
\hline
\end{tabular}


Table 1 (Continuation)

\begin{tabular}{|c|c|c|c|c|c|c|c|c|c|}
\hline Study & Needle & $\begin{array}{l}\text { Sam- } \\
\text { ple } \\
\text { size }\end{array}$ & $\begin{array}{l}\text { Study peri- } \\
\text { od/design }\end{array}$ & Country & Age & $\begin{array}{l}\text { Gender } \\
\text { male }\end{array}$ & $\begin{array}{l}\text { Lesion size } \\
(\mathrm{cm})\end{array}$ & Location & ROSE \\
\hline \multicolumn{10}{|c|}{ Fork-Tip versus FNA } \\
\hline $\begin{array}{l}\text { El Chafic } \\
2017 \\
{[23]}\end{array}$ & $\begin{array}{l}\text { Shark- } \\
\text { Core } \\
\text { EchoTip } \\
\text { Ultra/ } \\
\text { Expect }\end{array}$ & $\begin{array}{l}15 \\
91\end{array}$ & $\begin{array}{l}2011 \text { - } \\
\text { 2016/Ret- } \\
\text { rospective }\end{array}$ & USA & $\begin{array}{l}65 \pm 12.7 \\
64.8 \pm 15.7\end{array}$ & $\begin{array}{c}9(60 \%) \\
44(48.3 \%)\end{array}$ & $\begin{array}{l}2.5 \pm 0.9 \\
2.8 \pm 1.65\end{array}$ & $\begin{array}{l}\text { SE: } 100 \% \\
\text { SE: } 100 \%\end{array}$ & Yes \\
\hline $\begin{array}{l}\text { Jovani } \\
2017 \\
{[24]}\end{array}$ & $\begin{array}{l}\text { Shark- } \\
\text { Core } \\
\text { FNA }\end{array}$ & $\begin{array}{l}51 \\
51\end{array}$ & $\begin{array}{l}2015- \\
\text { 2016/Ret- } \\
\text { rospective }\end{array}$ & USA & $\begin{array}{l}63.5 \pm 12.7 \\
62.1 \pm 11.6\end{array}$ & $\begin{array}{l}22(43.1 \%) \\
27(52.9 \%)\end{array}$ & $\begin{array}{l}3.16 \pm 1.8 \\
2.67 \pm 1.1\end{array}$ & $\begin{array}{l}\text { Pancreas: } 57 \% \\
\text { Pancreas: } 57 \%\end{array}$ & NR \\
\hline $\begin{array}{l}\text { Kandel } \\
2016 \\
{[25]}\end{array}$ & $\begin{array}{l}\text { Shark- } \\
\text { Core } \\
\text { FNA }\end{array}$ & $\begin{array}{r}39 \\
117\end{array}$ & $\begin{array}{l}2012 \text { - } \\
\text { 2015/Ret- } \\
\text { rospective }\end{array}$ & USA & $\begin{array}{l}66(26-85) \\
70(17.91)\end{array}$ & $\begin{array}{l}19(49 \%) \\
65(56 \%)\end{array}$ & $\begin{array}{l}1.8(0.2-20) \\
2.3(0.4-11)\end{array}$ & $\begin{array}{l}\text { Pancreas: } 56 \% \\
\text { Pancreas: } 56 \%\end{array}$ & NR \\
\hline $\begin{array}{l}\text { Naveed } \\
2018 \\
{[26]}\end{array}$ & $\begin{array}{l}\text { Shark- } \\
\text { Core } \\
\text { EchoTip }\end{array}$ & $\begin{array}{l}115 \\
973\end{array}$ & $\begin{array}{l}2009- \\
\text { 2015/Ret- } \\
\text { rospective }\end{array}$ & USA & $\begin{array}{l}66.1 \\
66.7\end{array}$ & $\begin{array}{r}57(50 \%) \\
496(51 \%)\end{array}$ & $\begin{array}{l}2.75 \\
2.59\end{array}$ & $\begin{array}{l}\text { Pancreas: } \\
100 \% \\
\text { Pancreas: } \\
100 \%\end{array}$ & Yes \\
\hline $\begin{array}{l}\text { Song } \\
2018 \\
{[27]}\end{array}$ & $\begin{array}{l}\text { Shark- } \\
\text { Core }^{\circledR} \\
\text { Echo- } \\
\text { Tip/Ex- } \\
\text { pect }\end{array}$ & $\begin{array}{r}139 \\
42\end{array}$ & $\begin{array}{l}2013- \\
2017 / \text { Ret- } \\
\text { rospective }\end{array}$ & USA & $\begin{array}{l}64.7 \pm 11.9 \\
61.2 \pm 12.6\end{array}$ & $\begin{array}{l}47.5 \% \\
54.8 \%\end{array}$ & $\begin{array}{l}2.8 \pm 1.44 \\
2.8 \pm 1.8\end{array}$ & $\begin{array}{l}\text { Pancreas: } 78 \% \\
\text { Pancreas:79\% }\end{array}$ & No \\
\hline $\begin{array}{l}\text { Witt } \\
2018 \\
{[28]}\end{array}$ & $\begin{array}{l}\text { Shark- } \\
\text { Core } \\
\text { EchoTip }\end{array}$ & $\begin{array}{l}10 \\
10\end{array}$ & $\begin{array}{l}2015- \\
2016 / \text { Ret- } \\
\text { rospective }\end{array}$ & USA & NR & NR & NR & $\begin{array}{l}\text { Pancreas: } \\
100 \% \\
\text { Pancreas: } \\
100 \%\end{array}$ & Yes \\
\hline \multicolumn{10}{|c|}{ Fork-Tip versus Reverse bevel } \\
\hline $\begin{array}{l}\text { Abdelfa- } \\
\text { tah } 2018 \\
{[29]}\end{array}$ & $\begin{array}{l}\text { Shark- } \\
\text { Core } \\
\text { ProCore }\end{array}$ & $\begin{array}{l}162 \\
139\end{array}$ & $\begin{array}{l}2014- \\
\text { 2016/Ret- } \\
\text { rospective }\end{array}$ & USA & $\begin{array}{l}67 \pm 12 \\
67 \pm 11.8\end{array}$ & $\begin{array}{l}70(49 \%) \\
65(47 \%)\end{array}$ & $\begin{array}{l}2.5 \pm 1.4 \\
2.4 \pm 1.3\end{array}$ & $\begin{array}{l}\text { Pancreas: } 66 \% \\
\text { Pancreas: } 63 \%\end{array}$ & $\begin{array}{l}77 \% \\
98.3 \%\end{array}$ \\
\hline $\begin{array}{l}\text { Nayar } \\
2016 \\
{[30]}\end{array}$ & $\begin{array}{l}\text { Shark- } \\
\text { Core } \\
\text { ProCore }\end{array}$ & $\begin{array}{l}101 \\
100\end{array}$ & $\begin{array}{l}2013 \text { - } \\
\text { 2015/Ret- } \\
\text { rospective }\end{array}$ & UK & $\begin{array}{l}66.4 \\
68.1\end{array}$ & $\begin{array}{l}58(59 \%) \\
49(49 \%)\end{array}$ & $\begin{array}{l}3.4(1.4-9) \\
3.3(1-8.5)\end{array}$ & $\begin{array}{l}\text { Pancreas: } \\
100 \% \\
\text { Pancreas: } \\
100 \%\end{array}$ & No \\
\hline \multicolumn{10}{|c|}{ Franseen/Fork-Tip versus FNA } \\
\hline $\begin{array}{l}\text { Bang } \\
2019 \\
{[31]}\end{array}$ & $\begin{array}{l}\text { Ac- } \\
\text { quire/ } \\
\text { Shark- } \\
\text { Core } \\
\text { Expect }\end{array}$ & $\begin{array}{r}938 \\
2082\end{array}$ & $\begin{array}{l}2014 \text { - } \\
2017 / \text { Ret- } \\
\text { rospective }\end{array}$ & USA & $\begin{array}{l}67.1 \pm 12.9 \\
65.8 \pm 13.7\end{array}$ & $\begin{array}{c}510(54.4 \%) \\
1181(56 \%)\end{array}$ & $\begin{array}{l}2.88 \pm 1.32 \\
2.69 \pm 1.39\end{array}$ & $\begin{array}{l}\text { Pancreas: } 73 \% \\
\text { Nodes: } 9.4 \% \\
\text { Pancreas: } 71 \% \\
\text { Nodes: } 13 \%\end{array}$ & Yes \\
\hline \multicolumn{10}{|c|}{ Franseen versus Fork-Tip } \\
\hline $\begin{array}{l}\text { Abdelfa- } \\
\text { tah } 2018 \\
{[32]}\end{array}$ & $\begin{array}{l}\text { Acquire } \\
\text { Shark- } \\
\text { Core }\end{array}$ & $\begin{array}{l}97 \\
97\end{array}$ & $\begin{array}{l}2015- \\
2017 / \text { Ret- } \\
\text { rospective }\end{array}$ & USA & $\begin{array}{l}63.7 \pm 10.8 \\
62.8 \pm 15.5\end{array}$ & $\begin{array}{l}47 \% \\
52 \%\end{array}$ & $\begin{array}{l}2.4 \pm 1.3 \\
2.5 \pm 1.4\end{array}$ & $\begin{array}{l}\text { Pancreas: } 55 \% \\
\text { Pancreas: } 48 \%\end{array}$ & $14.5 \%$ \\
\hline $\begin{array}{l}\text { Bang } \\
2018 \\
{[33]}\end{array}$ & $\begin{array}{l}\text { Acquire } \\
\text { Shark- } \\
\text { Core }\end{array}$ & $\begin{array}{l}50 \\
50\end{array}$ & $\begin{array}{l}2016 \text { - } \\
2017 / \text { Cross- } \\
\text { over RCT }\end{array}$ & USA & $71.3 \pm 11$ & $28(56 \%)$ & $2.4 \pm 0.6$ & $\begin{array}{l}\text { Pancreas: } \\
100 \%\end{array}$ & Yes \\
\hline
\end{tabular}


- Table 2 Overall and subgroup analysis of main diagnostic outcomes. Subgroup analysis was performed based on a) location of the target lesion (pancreas, nodes, subepithelial lesion), and b) needle used (Franseen versus Fork-Tip). Numbers in parentheses indicate $95 \%$ confidence intervals.

\begin{tabular}{|c|c|c|c|c|c|}
\hline Location & Needle & No. of Cohorts & No. of patients & $\begin{array}{l}\text { Summary Estimate } \\
(95 \% \mathrm{Cl})\end{array}$ & $\begin{array}{l}\text { Within-group } \\
\text { heterogeneity }\left(\mathrm{I}^{2}\right)\end{array}$ \\
\hline \multicolumn{6}{|l|}{ Sample Adequacy } \\
\hline \multirow{3}{*}{ Overall } & Overall & 25 & 2894 & $94.8 \%(93.1 \%-96.4 \%)$ & $87 \%$ \\
\hline & Franseen & 12 & 857 & $96.1 \%(93.7 \%-98.4 \%)$ & $82.9 \%$ \\
\hline & Fork-Tip & 13 & 1099 & $92.4 \%(88.8 \%-96 \%)$ & $88 \%$ \\
\hline \multirow{3}{*}{ Pancreas } & Overall & 22 & 1987 & $95.6 \%(94 \%-97.3 \%)$ & $48.9 \%$ \\
\hline & Franseen & 10 & 567 & $97 \%(94.8 \%-99.3 \%)$ & $13.4 \%$ \\
\hline & Fork-Tip & 11 & 736 & $92.6 \%(88.8 \%-96.4 \%)$ & $34 \%$ \\
\hline \multirow{3}{*}{ Nodes } & Overall & 8 & 223 & $97.2 \%(94 \%-100 \%)$ & $52.6 \%$ \\
\hline & Franseen & 4 & 95 & $98.4 \%(96 \%-100 \%)$ & $0 \%$ \\
\hline & Fork-Tip & 3 & 54 & $77.6 \%(46.2 \%-100 \%)$ & $39 \%$ \\
\hline \multirow{3}{*}{ Subepithelial Lesion } & Overall & 9 & 218 & $95.6 \%(92.2 \%-98.9 \%)$ & $27 \%$ \\
\hline & Franseen & 5 & 68 & $97.6 \%(94 \%-100 \%)$ & $0 \%$ \\
\hline & Fork-Tip & 4 & 64 & $90.5 \%(79.6 \%-100 \%)$ & $38.9 \%$ \\
\hline \multicolumn{6}{|c|}{ Histological Core Procurement } \\
\hline \multirow{3}{*}{ Overall } & Overall & 17 & 1932 & $92.5 \%(89.8 \%-95.3 \%)$ & $79.8 \%$ \\
\hline & Franseen & 7 & 455 & $93.5 \%(89.8 \%-97.2 \%)$ & $65 \%$ \\
\hline & Fork-Tip & 9 & 539 & $90.8 \%(85.5 \%-96.2 \%)$ & $84 \%$ \\
\hline \multirow{3}{*}{ Pancreas } & Overall & 11 & 1192 & $93.7 \%(90.7 \%-96.8 \%)$ & $35.3 \%$ \\
\hline & Franseen & 5 & 225 & $94 \%(89 \%-99 \%)$ & $36.3 \%$ \\
\hline & Fork-Tip & 5 & 283 & $93.1 \%(87.3 \%-99 \%)$ & $33.8 \%$ \\
\hline \multicolumn{6}{|l|}{ Diagnostic Accuracy } \\
\hline \multirow{3}{*}{ Overall } & Overall & 15 & 945 & $95 \%(93.5 \%-96.5 \%)$ & $11 \%$ \\
\hline & Franseen & 8 & 492 & $95 \%(92.5 \%-97.5 \%)$ & $37 \%$ \\
\hline & Fork-Tip & 7 & 453 & $94.4 \%(92.3 \%-96.5 \%)$ & $0 \%$ \\
\hline \multirow{3}{*}{ Pancreas } & Overall & 11 & 631 & $96.2 \%(94.7 \%-97.7 \%)$ & $0 \%$ \\
\hline & Franseen & 7 & 324 & $96.8 \%(94.9 \%-98.7 \%)$ & $0 \%$ \\
\hline & Fork-Tip & 4 & 307 & $95.2 \%(92.8 \%-97.6 \%)$ & $0 \%$ \\
\hline \multicolumn{6}{|l|}{ Diagnostic Sensitivity } \\
\hline \multirow{3}{*}{ Overall } & Overall & 10 & 755 & $92.8 \%(89.8 \%-95.7 \%)$ & $64.2 \%$ \\
\hline & Franseen & 4 & 255 & $93.3 \%(89.2 \%-97.4 \%)$ & $45 \%$ \\
\hline & Fork-Tip & 6 & 500 & $92.2 \%(87.9 \%-96.6 \%)$ & $73 \%$ \\
\hline \multirow{3}{*}{ Pancreas } & Overall & 6 & 445 & $94.1 \%(90.7 \%-97.6 \%)$ & $40.4 \%$ \\
\hline & Franseen & 2 & 81 & $95.3 \%(90.7 \%-99.9 \%)$ & $0 \%$ \\
\hline & Fork-Tip & 4 & 364 & $93.4 \%(88.3 \%-98.4 \%)$ & $45.6 \%$ \\
\hline
\end{tabular}

$\mathrm{Cl}$, confidence interval.

The study by Bang et al [31] did not report a subgroup analysis based on the needle design, therefore data from this study were considered only in the overall analysis. 


\section{Studies}

Abdelfatah 2018

Adler 2018

Bang 2018

Bang 2017

Bang (b) 2017

Haseeb 2018

Ishikawa 2019

Leung Ki 2019

Mukai 2018

Sugiura 2019

Overall $\left(I^{2}=13.43 \%\right)$

a

\section{Studies}

Abdelfatah (b) 2018

DiMaio 2016

Jovani 2017

Kandel 2016

Larsen 2018

Naveed 2018

Nayar 2016

Song 2018

Witt 2018

Abdelfatah II 2018

Bang II 2018

Overall $\left(I^{2}=34 \%\right)$

b
Estimate $(95 \% \mathrm{Cl}) \quad \mathrm{Ev} / \mathrm{Trt}$

$\begin{array}{lr}0.642(0.512,0.771) & 34 / 53 \\ 0.982(0.956,1.000) & 107 / 109 \\ 0.940(0.874,1.000) & 47 / 50 \\ 0.944(0.839,1.000) & 17 / 18 \\ 0.989(0.960,1.000) & 46 / 46 \\ 0.995(0.981,1.000) & 99 / 99 \\ 0.990(0.963,1.000) & 50 / 50 \\ 0.984(0.941,1.000) & 31 / 31 \\ 0.984(0.940,1.000) & 30 / 30 \\ 0.951(0.903,0.998) & 77 / 81\end{array}$

$0.970(0.948,0.993) \quad 538 / 567$
Estimate $(95 \% \mathrm{Cl}) \quad$ Ev/Trt

$0.776(0.697,0.855) \quad 83 / 107$

$0.802(0.726,0.878) \quad 85 / 106$

$0.983(0.938,1.000) \quad 29 / 29$

$0.978(0.919,1.000) \quad 22 / 22$

$0.976(0.928,1.000) \quad 40 / 41$

$0.939(0.895,0.983) \quad 108 / 115$

$0.990(0.971,1.000) \quad 100 / 101$

$0.870(0.807,0.934) \quad 94 / 108$

$0.955(0.831,1.000) \quad 10 / 10$

$0.851(0.749,0.953) \quad 40 / 47$

$0.980(0.941,1.000) \quad 49 / 50$

$0.926(0.888,0.964) \quad 660 / 736$

0.7

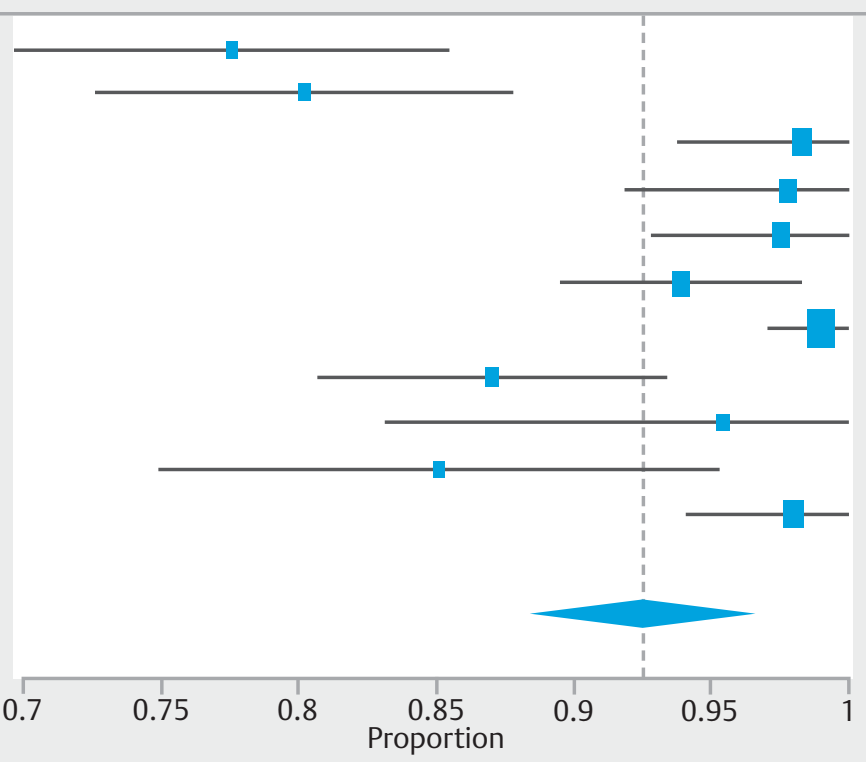

- Fig. 2 Pooled analysis assessing rates of sample adequacy of a Franseen and $\mathbf{b}$ Fork-tip fine-needle biopsy in targeting pancreatic lesions. Sample adequacy in targeting pancreatic masses was $95.6 \%(94 \%-97.3 \% ; 12=48.9 \%)$ with significantly higher rates of adequate samples obtained with Franseen needle ( $97 \%, 94.8 \%$ to $99.3 \%$ versus $92.6 \%, 88.8 \%$ to $96.4 \%$; $P=0.006)$.

\section{Number of passes and adverse events}

Analysis of number of needle passes needed to obtain adequate sample showed favorable results with the new needles in comparison to standard FNA (mean difference: $-0.42,-0.94$ to 0.09 , and $-1.60,-2.23$ to -0.98 with Franseen and Fork-tip needle, respectively; Supplementary Fig.6).

Both needles were significantly superior to FNA when restricting the analysis to pancreatic masses $(-0.44,-0.87$ to -0.01 , and $-1.82,-2.20$ to -1.43 with the two needles, respectively; Fig. 3). Heterogeneity was moderate to high $\left(\mathrm{I}^{2}=48 \%\right.$ to $92 \%$ ) and no evidence of publication bias was detected.
Details on safety profile of the two devices are reported in Supplementary Table 5. Of note, only a small number of patients experienced adverse events and all of these complications (mainly bleeding) were mild and did not impact on patient outcomes.

\section{Discussion}

EUS-TA plays a pivotal role in the diagnostic algorithm of solid masses but its diagnostic accuracy is strictly dependent on a series of lesion-related features (such as size, number, histological type) and technical variables like needles adopted, number of 


\begin{tabular}{|c|c|c|c|c|c|c|c|c|c|c|}
\hline \multirow[b]{2}{*}{ Study or subgroup } & \multicolumn{3}{|c|}{ New needle } & \multicolumn{2}{|c|}{ FNA } & \multirow[b]{2}{*}{ Total } & \multirow[b]{2}{*}{ Weight } & \multirow{2}{*}{$\begin{array}{l}\text { Mean difference } \\
\text { IV, random, } 95 \% \mathrm{CI}\end{array}$} & \multirow{2}{*}{\multicolumn{2}{|c|}{$\begin{array}{c}\text { Mean difference } \\
\text { IV, random, } 95 \% \mathrm{CI}\end{array}$}} \\
\hline & Mean & SD & Total & Mean & SD & & & & & \\
\hline Bang 2017 & 1.15 & 0.47 & 46 & 1.18 & 0.58 & 46 & $26.2 \%$ & $-0.03[-0.25,0.19]$ & 1 & \\
\hline Bang 2019 & 1.9 & 1.5 & 938 & 2.5 & 1.8 & 2082 & $27.4 \%$ & $-0.60[-0.72,-0.48]$ & 4 & \\
\hline Ishikawa 2019 & 2 & 1 & 50 & 2 & 1 & 36 & $22.0 \%$ & $0.00[-0.43,0.43]$ & 1 & \\
\hline Mukai 2018 & 2.1 & 0.4 & 30 & 3.2 & 0.8 & 30 & $24.3 \%$ & $-1.10[-1.42,-0.78]$ & 4 & \\
\hline \multicolumn{2}{|l|}{ Total $(95 \% \mathrm{Cl})$} & & 1064 & & & 2194 & $100.0 \%$ & $-0.44[-0.87,-0.01]$ & $i$ & \\
\hline \multicolumn{8}{|c|}{ Heterogeneity: Tau $^{2}=0.17 ; \mathrm{Chi}^{2}=39.49, \mathrm{df}=3(P<0.00001) ; \mathrm{I}^{2}=92 \%$} & -100 & 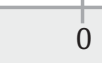 & 50 \\
\hline \multicolumn{8}{|c|}{ Test for overall effect: $Z=1.99(P=0.05)$} & Favou & & Favours FNA \\
\hline
\end{tabular}

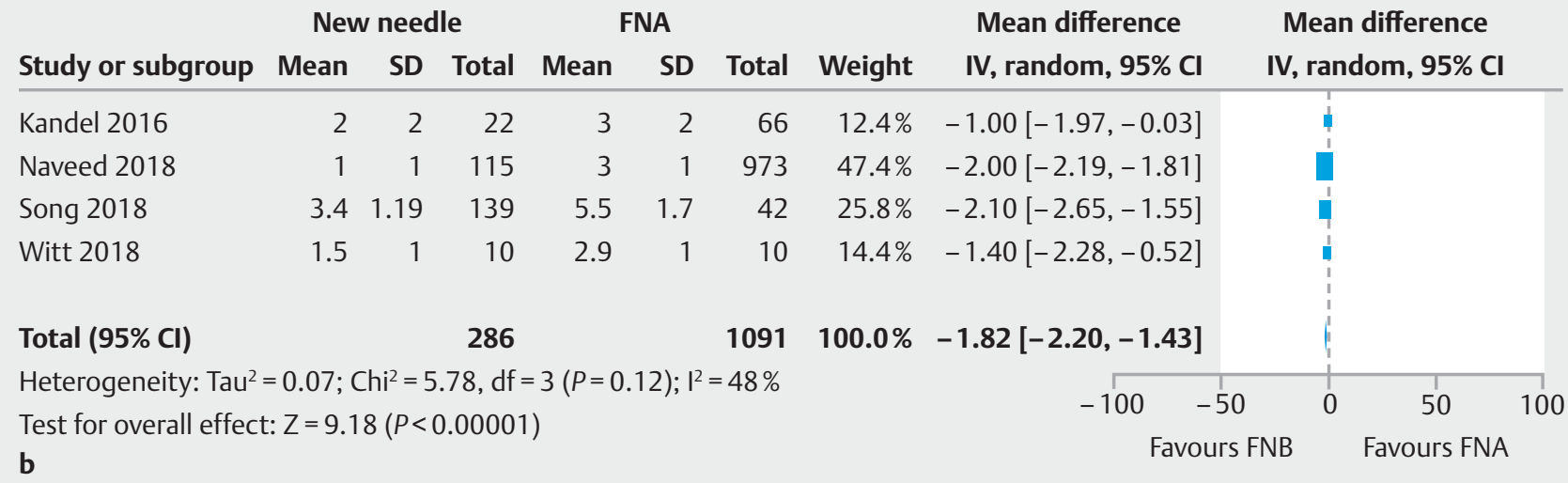

- Fig. 3 Meta-analysis comparing mean number of needle passes of a Franseen and $\mathbf{b}$ Fork-tip needle in comparison to fine-needle aspiration. Both needles resulted in significantly superior to fine-needle aspiration (FNA) when restricting the analysis to pancreatic masses (-0.44, -0.87 to -0.01 , and $-1.82,-2.20$ to -1.43 with the two needles, respectively). Heterogeneity was moderate to high $\left(\mathrm{I}^{2}=48 \%\right.$ to $\left.92 \%\right)$.

passes or availability of ROSE. To at least partially overcome these limitations and given the pressing need for adequate histological samples for molecular analysis, biopsy needles have been developed and introduced in clinical practice [34].

Given recent development of novel FNB needle designs (such as Franseen and Fork-tip needles), there is a clear need to systematically evaluate the impact of these newer devices on EUS-TA. The current manuscript represents the first attempt to systematically assess diagnostic performance of newer FNB needles, namely Franseen and Fork-tip needles, in a variety of abdominal masses.

With a meta-analysis of 24 studies selectively evaluating Franseen and Fork-tip needles (alone or in comparison to a control group), we made several key observations.

First, the two newer needles showed striking results in terms of sample adequacy (94.8\%), rate of histological optimal core procurement (92.5\%), diagnostic accuracy (95\%), and sensitivity $(92.8 \%)$. Although a significant increase in sample adequacy was observed with Franseen needle as compared to Fork-tip (96.1\% versus $92.4 \%, P<0.001$ ), the other diagnostic outcomes were perfectly comparable between the two needle designs with no significant difference observed in terms of histological core procurement, diagnostic accuracy, or sensitivity. Second, all sensitivity analyses confirmed findings from the main analy- sis, in particular both Franseen and Fork-tip needles performed well in targeting all abdominal lesions (sample adequacy $95.6 \%$ for pancreatic masses, $97.2 \%$ for lymph nodes, and $95.6 \%$ for SELs). As expected, use of ROSE did not impact significantly on diagnostic yield. Third, meta-analysis of head-to-head studies directly comparing FNB to FNA showed a clear advantage in terms of sample adequacy of both FNB needles $(P=0.007$ and $P=0.05$, respectively). Fourth, as already pointed out in previous meta-analyses $[3,4]$, FNB required a lower number of needle passes through the lesion to obtain adequate samples in comparison to standard FNA. Fifth, both FNB needles resulted to be safe with only a small number of patients experiencing mainly mild pancreatitis or bleeding events.

These findings, which are considerably more favorable as compared to those reported in previous meta-analyses on a reverse bevel FNB needle [3], are likely to be related to the different design of newer needles with higher number of cutting points ( 3 in Franseen and 6 in Fork-tip needle) designed to provide improved control at the puncture site and stability at the tip, allowing for enhanced penetration [35]. As tissue moves into the tip of the Franseen needle, suction is applied to push the tissue into three symmetrical cutting heels which cut tissue from three different angles to make a more circular cut. In the case of the Fork-tip device, two opposing catch beveled tips al- 
low funneling of target tissue into the lumen of the needle, while a distal cutting design minimizes stacking and fracturing of collected samples to produce more intact tissue architecture [35].

As expected, the aforementioned results were not influenced by use of ROSE, a tool not routinely adopted in nonAmerican centers, thus confirming that FNB may obviate the need for an on-site pathologist to obtain optimal diagnostic outcomes. Moreover, increased costs of newer needles may be at least partially compensated for by obviating the need for an on-site pathologist.

Because most of the included studies were single-cohort non-comparative series, results regarding the higher sample adequacy observed with the Franseen needle as compared to the Fork-tip device should be interpreted with caution, considering that the single head-to-head trial directly comparing the two newer FNB needles did not report significant differences between the two devices [15].

There are some limitations to our study. First, the limited number of case-control or randomized studies does not allow a strong comparison between newer needles and standard FNA or reverse bevel FNB. This aspect requires particular caution in interpreting our comparative findings due to the high risk of indirectness. In fact, the validity of indirect comparisons rests on the assumption that factors in the design of the included studies (patients, co-interventions, measurement of outcomes) and methodological quality are not sufficiently different to result in different effects (the so-called "similarity assumption" [36]). Because this assumption is always in some doubt, indirect comparisons always warrant rating down by one level in quality of evidence. Second, as most included studies used 22G needles, subanalysis based on needle caliper was not feasible. Therefore, absent of studies specifically testing different calipers, our results should be considered applicable only to the 22G FNB device, which is indeed the most frequently used FNB needle worldwide.

\section{Conclusion}

In conclusion, despite these weaknesses, our meta-analysis speaks in favor of use of newer biopsy needles as safe and effective tools in EUS-guided tissue acquisition. In particular, based on the extremely high rates of optimal histological core procurement, they may represent a valuable option in conditions that require assessment of tissue architecture (for example, in oncologic studies that require core biopsies for personalized medicine or benign conditions such as autoimmune pancreatitis). Further RCTs comparing the two newer needles to reverse bevel FNB or each other are warranted to confirm these promising results.

\section{Competing interests}

None

\section{References}

[1] Wani S, Mullady D, Early DS et al. The clinical impact of immediate onsite cytopathology evaluation during endoscopic ultrasound-guided fine needle aspiration of pancreatic masses: a prospective multicenter randomized controlled trial. Am J Gastroenterol 2015; 110: 1429 1439

[2] Wani S, Muthusamy VR, McGrath CM et al. AGA White Paper: Optimizing Endoscopic Ultrasound-Guided Tissue Acquisition and Future Directions. Clin Gastroenterol Hepatol 2018; 16: 318 - 327

[3] Bang JY, Hawes R, Varadarajulu S. A meta-analysis comparing ProCore and standard fine-needle aspiration needles for endoscopic ultrasound-guided tissue acquisition. Endoscopy 2016; 48: 339 - 349

[4] Facciorusso A, Singh Bajwa H, Menon K et al. Comparison between 22-gauge aspiration and 22-gauge biopsy needles for eus-guided sampling of pancreatic lesions: a meta-analysis. Endosc Ultrasound 2019: doi:10.4103/eus.eus_4_19

[5] Higgins JP, Altman DG, Gøtzsche PC et al. The Cochrane Collaboration's tool for assessing risk of bias in randomised trials. BMJ 2011; 343: d5928

[6] Wells GA, Shea B, O'Connell D et al. The Newcastle - Ottawa Scale (NOS) for assessing the quality of nonrandomised studies in metaanalyses. Available from: http://www.ohri.ca/programs/ clinical_ epidemiology/oxford.htm, accessed on March 2019

[7] Reitsma JB, Glas AS, Rutjes AW et al. Bivariate analysis of sensitivity and specificity produces informative summary measures in diagnostic reviews. J Clin Epidemiol 2005; 58: $982-990$

[8] Shah ND, Sasatomi E, Baron TH. Endoscopic Ultrasound-guided Parenchymal Liver Biopsy: Single Center Experience of a New Dedicated Core Needle. Clin Gastroenterol Hepatol 2017; 15: 784-786

[9] Bekkali NLH, Nayar MK, Leeds JS et al. Impact of metal and plastic stents on endoscopic ultrasound-guided aspiration cytology and core histology of head of pancreas masses. Endoscopy 2019: doi:10.1055/ a-0824-6982

[10] Adler DG, Muthusamy VR, Ehrlich DS et al. A multicenter evaluation of a new EUS core biopsy needle: Experience in 200 patients. Endosc UItrasound 2019; 8: $99-104$

[11] Bang JY, Hebert-Magee S, Hasan MK et al. Endoscopic ultrasonography-guided biopsy using a Franseen needle design: Initial assessment. Dig Endosc 2017; 29: 338-346

[12] Haseeb A, Taylor LJ, Adler DG. Comparing endoscopic ultrasoundguided core biopsies of solid pancreatic and extrapancreatic lesions: a large single-operator experience with a new fine-needle biopsy needle. Ann Gastroenterol 2018; 31: 742 - 746

[13] Leung Ki EL, Lemaistre Al, Fumex F et al. Macroscopic onsite evaluation using endoscopic ultrasound fine needle biopsy as an alternative to rapid onsite evaluation. Endosc Int Open 2019; 7: E189-E194

[14] Sugiura R, Kuwatani M, Yane K et al. Prospective, multicenter, observational study of tissue acquisition through EUS-guided fine-needle biopsy using a 25G Franseen needle. Endosc Ultrasound 2019: doi:10.4103/eus.eus_66_18

[15] Bang JY, Hebert-Magee S, Navaneethan U et al. Randomized trial comparing the Franseen and Fork-tip needles for EUS-guided fineneedle biopsy sampling of solid pancreatic mass lesions. Gastrointest Endosc 2018; 87: $1432-1438$

[16] El Hajj II, Wu H, Reuss S et al. Prospective Assessment of the Performance of a New Fine Needle Biopsy Device for EUS-Guided Sampling of Solid Lesions. Clin Endosc 2018; 51: 576-583

[17] Fujita A, Ryozawa S, Kobayashi M et al. Diagnostic ability of a $22 \mathrm{C}$ Franseen needle in endoscopic ultrasound-guided fine needle aspiration of subepithelial lesions. Mol Clin Oncol 2018; 9: 527 - 531

[18] Ishikawa T, Kawashima H, Ohno E et al. Clinical Impact of EUS-Guided Fine Needle Biopsy Using a Novel Franseen Needle for Histological 
Assessment of Pancreatic Diseases. Can J Gastroenterol Hepatol 2019; 2019: 8581743

[19] Mukai S, Itoi T, Yamaguchi H et al. A retrospective histological comparison of EUS-guided fine-needle biopsy using a novel franseen needle and a conventional end-cut type needle. Endosc Ultrasound 2019; 8: $50-57$

[20] DiMaio C], Kolb JM, Benias PC et al. Initial experience with a novel EUSguided core biopsy needle (SharkCore): results of a large North American multicenter study. Endosc Int Open 2016; 4: E974- 979

[21] Ishikawa T, Mohamed R, Heitman S] et al. Diagnostic yield of small histological cores obtained with a new EUS-guided fine needle biopsy system. Surg Endosc 2017; 31: 5143 - 5149

[22] Larsen MH, Fristrup CW, Detlefsen $S$ et al. Prospective evaluation of EUS-guided fine needle biopsy in pancreatic mass lesions. Endosc Int Open 2018; 6: E242 -E248

[23] El Chafic AH, Loren D, Siddiqui A et al. Comparison of FNA and fineneedle biopsy for EUS-guided sampling of suspected GI stromal tumors. Gastrointest Endosc 2017; 86: 510-515

[24] Jovani M, Abidi WM, Lee LS. Novel fork-tip needles versus standard needles for EUS-guided tissue acquisition from solid masses of the upper GI tract: a matched cohort study. Scand J Gastroenterol 2017; 52: $784-787$

[25] Kandel P, Tranesh G, Nassar A et al. EUS-guided fine needle biopsy sampling using a novel fork-tip needle: a case-control study. Gastrointest Endosc 2016; 84: 1034 - 1039

[26] Naveed M, Siddiqui AA, Kowalski TE et al. A Multicenter comparative trial of a novel EUS-guided core biopsy needle (SharkCore ${ }^{\mathrm{TM}}$ ) with the 22-gauge needle in patients with solid pancreatic mass lesions. Endosc Ultrasound 2018; 7: 34-40

[27] Song Z, Trujillo CN, Song H et al. Endoscopic Ultrasound-Guided Tissue Acquisition Using Fork-Tip Needle Improves Histological Yield,
Reduces Needle Passes, Without On-Site Cytopathological Evaluation. J Pancreat Cancer 2018; 4: 75-80

[28] Witt BL, Factor RE, Chadwick BE et al. Evaluation of the SharkCore ${ }^{\circledR}$ needle for EUS-guided core biopsy of pancreatic neuroendocrine tumors. Endosc Ultrasound 2018; 7: $323-328$

[29] Abdelfatah MM, Hamed A, Koutlas NJ et al. The diagnostic and cellularity yield of reverse bevel versus fork-tip fine needle biopsy. Diagn Cytopathol 2018: doi:10.1002/dc.23966

[30] Nayar MK, Paranandi B, Dawwas MF et al. Comparison of the diagnostic performance of 2 core biopsy needles for EUS-guided tissue acquisition from solid pancreatic lesions. Gastrointest Endosc 2017; 85: $1017-1024$

[31] Bang JY, Kirtane S, Krall K et al. In memoriam - fine needle aspiration, Birth - fine needle biopsy:The changing trend in endoscopic ultrasound-guided tissue acquisition. Dig Endosc 2018: doi:10.1111/ den. 13280

[32] Abdelfatah MM, Grimm IS, Gangarosa LM et al. Cohort study comparing the diagnostic yields of 2 different EUS fine-needle biopsy needles. Gastrointest Endosc 2018; 87: 495-500

[33] Bang JY, Hebert-Magee S, Navaneethan U et al. EUS-guided fine needle biopsy of pancreatic masses can yield true histology: results of a randomised trial. Gut 2017; 67: 2081-2084

[34] Facciorusso A, Buccino RV, Muscatiello N. How to measure quality in endoscopic ultrasound. Ann Transl Med 2018; 6: 266

[35] James TW, Baron TH. A comprehensive review of endoscopic ultrasound core biopsy needles. Expert Rev Med Devices 2018; 15: 127 135

[36] Song F, Loke YK, Walsh T et al. Methodological problems in the use of indirect comparisons for evaluating healthcare interventions: survey of published systematic reviews. BMJ 2009; 338: b1147 